\title{
Real-Time Detection of Knitting Fabric Defects Using Shearlet Transform
}

\author{
Kazım Hanbay $^{1 *}$, Muhammed Fatih Talu², Ömer Faruk Özgüven ${ }^{3}$,Dursun Öztürk ${ }^{4}$ \\ ${ }^{1}$ Bingol University, Department of Computer Engineering, Selahadn-i Eyyubi Mah. Aydınlık Cad. No:1 Bingöl / Turkey \\ ${ }^{2}$ Inonu University, Department of Computer Engineering, 44280, Malatya, Turkey \\ ${ }^{3}$ Inonu University, Department of Biomedical Engineering, 44280, Malatya, Turkey \\ ${ }^{4}$ Bingol University, Department of Electrical-Electronic Engineering, 12000, Bingol, Turkey
}

Corresponding Author: Kazım HANBAY, khanbay@bingol.edu.tr.

\begin{abstract}
This paper proposes a vision-based fabric inspection system for the circular knitting machine. Firstly, a comprehensive fabric database called Fabric Defect Detection Database (FDDD) are constructed. To extract significant features of fabric images, shearlet transform is used. Means and variances are calculated from all subbands and combined into a high-dimensional feature vector. The proposed system is evaluated on a circular knitting machine in a textile factory. The real-time performance analysis is only carried out by inspecting single jersey knitted fabric. Our proposed system achieves the highest accuracy of $94.0 \%$ in the detection of single jersey knitting fabric defects.
\end{abstract}

\author{
ARTICLE HISTORY \\ Received: 08.12.2017 \\ Accepted: 01.09.2018

\section{KEYWORDS} \\ Fabric defect detection, real- \\ time inspection, texture \\ classification
}

\section{INTRODUCTION}

Fabric defect detection is one of the most important problems in textile industry. There are approximately 70 identified categories of fabric defects defined by the textile industry [1]. In recent years, computer vision based fabric inspection systems are widely used as defect detection system. Current defect detection approaches can be classified as spatial domain approaches and frequency domain approaches. Spectral approaches are based on the periodicity of the texture images. In spatial approaches, spatial distribution of pixel gray values is extracted by different algorithms [1,2,3] such as co-gray level cooccurrence matrices (GLCM), Co-occurrence Histogram of Oriented Gradient (Co-HOG) and mathematical morphology. For example, co-occurrence matrix was used to extract six textural features for twill fabric defects [2]. To detect the woven fabrics defects, Gabor wavelet transform was used [4]. Firstly, texture features were extracted with the Gabor filter bank while varying the two parameters orientations and scales. Then, the mean and standard deviation of each filtered fabric images were calculated and taken as a feature vector. The frequency domain approaches generally use various mathematical transformations and auxiliary filters. Ismail et al. [5] evaluated the realities of fabric images using the Fourier spectrum. Hanmandlu et al. [6] implemented the topothesy and fractal dimension methods in fabric textures. $\mathrm{Li}$ et al. [7] developed direct thresholding method based on a wavelet transform. For broken-end defects on fabric surface, they obtained high detection rate. In [8], Tsai and Chiang considered the fabric images of oriented and nonoriented textures. They used different sub-bands of wavelet transform for finding the defects in fabric images. To highlight the defective area in the fabric image, Hu et al. [9] developed a wavelet shrinkage method. In another study, to identify eight different types of defects, adaptive wavelet transform was used. Karlekar et al. [10] applied wavelet transform for modelling of fabric texture and defect detection. The fabric image was decomposed up to two levels for defect detection. Then direct thresholding and filtering methods were used for obtaining final defective area for analysis. Satisfactory results were obtained on line defects. Defects classification was also performed using the neural networks. Feed forward neural network was used to

To cite this article: Hanbay K.., Talu M.F., Özgüven Ö.F.., Öztürk D.,2018, “Real-Time Detection of Knitting Fabric Defects Using Shearlet Transform” Tekstil ve Konfeksiyon, 29 (1), pp:3-10. 
detect fabric defects [11]. Twill and plain weave fabric defects were classified successfully. Only certain types of defects were detected through such wavelet transform and neural network such as strip bar, bamboo and wool ball [12]. Zhang et al. [13] developed a method for fabric defect classification using radial basis function (RBF) network. RBF network is a special kind of Artificial Neural Network (ANN), and it can have several radial basis functions such as Gaussian and inverse multi-quadrics function. In this work, they extracted the gray level arrangement in the neighborhood of each pixel as the feature. The RBF network was used as the classifier. The developed method was tested on fabric defect images with nine classes [13]. Recently, multi-scale geometric analysis (MGA) methods have been proposed such as Shearlet transform [14]. Discreate shearlet transform (DST) can be adaptively used to analyze data with anisotropic information at multiple scales. In order to reduce size of feature vectors, Principal Component Analysis (PCA) is introduced as a technique of dimension reduction [15]. Combined with PCA and discrete shearlet transform (DST), we propose a new feature extraction method called DST-PCA.

\section{FABRIC IMAGE ACQUISITION}

In this paper, a line-scan CCD camera has been used to capture the fabric images. Frontlighting method is used to capture higher contrast images. Line-scan camera has been installed on the circular knitting machine. Camera and linear light are fixed on the beam over the knitting machine, and their position adjusted a $90^{\circ}$ angle between auto focus lens and fabric surface. The resolution of the line-scan camera is 2048 pixels. The line rate of camera is $51 \mathrm{kHz}$, and it has 7 $\mu \mathrm{m} \times 7 \mu \mathrm{m}$ pixel size (Horizontal $\times$ Vertical). We use a 16 $\mathrm{mm}$ auto focus lens. The maximum image circle of lens is $2 / 3$ ". So the resolution of line-camera can be calculated as $2048 \times\left(\frac{2}{3}\right)=1365.33$ pixels. However, we have investigated that the width of inspected fabric is $80 \mathrm{~mm}$. The production speed of circular knitting machine is 25 RPM (Revolutions Per Minute), and diameter of machine is 20 inch. Thus, production per minute for single jersey circular knitting machine is $1.40 \mathrm{~m}$ approximately. The distance between line-scan camera and fabric surface is $90 \mathrm{~mm}$. In this paper, defects with a size bigger than $3 \mathrm{~mm}$ are detect. To ensure that $3 \mathrm{~mm}$ can be adequately resolved, this defects should be depicted on more than 37 pixels. Thus, each pixel of image is responsible for a distance of $3 \mathrm{~mm} / 37$
$=0.081 \mathrm{~mm}$. For the resolution of the fabric image obtained at calculated pixel resolution, we need $80 \mathrm{~mm} /(0.081 \mathrm{~mm} /$ pixel $) \approx 988$ pixels. To capture high resolution images, the resolution of linecamera is adjusted as 1000 pixels. The resolution of image height, which is the distance between two adjacent lines captured by the line-scan camera, must be equal to image width. As the height of an image is 256 pixels, the length of the fabric surface covered by an image is $256 \times 0.081=20.73 \mathrm{~mm}$. Using the fabric database we built earlier [1,16], we constructed a comprehensive textile texture database called Fabric Defect Detection Database (FDDD). This database consists of 12 video sequences. There are a total of 691 frames in the video sequences. Five different knitting fabric kinds were included in the database. Based on the analysis of fabric images, four defective classes were defined. To extend the database, the frames of each video sequences were divided into sub-images. The size of acquired images was $256 \times 1000$. For database construction, the window size was often fixed and assumes that sufficient area of the fabric image was represented to extend the database. Thus window size was chosen as $256 \times 50$. Moving window operation was only performed on columns of each frame and the frames were divided into sub-images. Thus, the size of sub-images was $256 \times 250$. The number of subimages for a single frame is calculated as follows:

$$
\text { Number of sub_images }=\frac{c}{w}
$$

where $C$ is the number of columns in frame and $w$ is the window size. For $C=1000$ and $w=50,20$ subimages were produced from each frame. As a result, the database consists

of 691 frame $\times 20$ sub_frame $=13820 \quad$ fabric images.

For the development of objective and reliable defect detection methods, this database will provide important contributions to the literature. Figure 1 shows four defective types of fabric images, including needle breakages, hole, press-off and gout. Needle breakage and hole are the most common defects. Gout is a lumpy defect and it occurs during spinning. Press-off is a knitting distortion in horizontal direction and vertical direction. a)

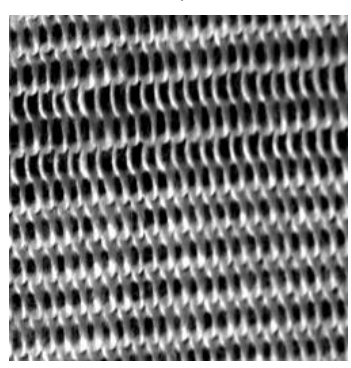

b)

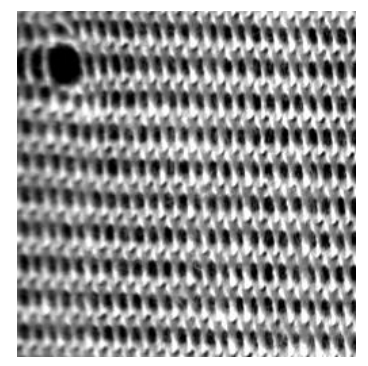

c)

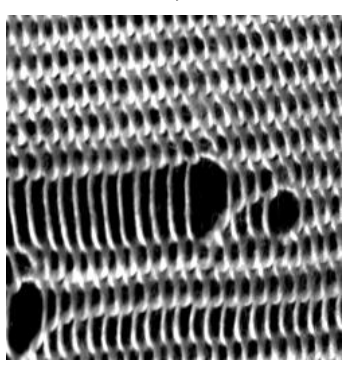

d)

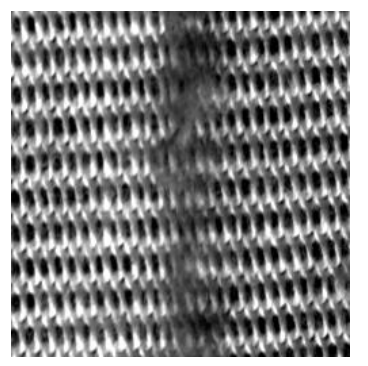

Figure 1. Images of fabric defects: (a) needle breakages, (b) holes, (c) press-off, (d) gout 


\section{METHODS OF FREQUENCY DOMAIN}

\subsection{Fourier Transform}

Fast Fourier Transform (FFT) can be applied to compare the spectrum of a fabric image containing a defect with that of a defect-free one [17]. The frequency domain is robust to characterize the fabric defects while the spatial domain is sensitive to noise. Therefore, FFT can be applied to compare the spectrum of a fabric image containing a defect with that of a defect-free one. When a fabric defect occurs, the periodic fabric pattern break downs. As a result of this situation, some specific positions of the frequency spectrum change which could signify the presence of a defect. These irregularities (i.e. defects) correspond to fabric defects. After the spectrum is calculated, many statistical features can be used to detect defects of fabrics. The statistical features provide enough textural information about fabric pattern [18]. In this paper, we used FFT to extract seven statistical features from the Fourier spectrum of the fabric images.

$f(x, y)$ is the gray level at pixel coordinates $(x, y)$ in the original fabric image of $M \times N$. For frequency variables $a, b$, the Discrete Fourier Transform $F(a, b)$ is defined by:

$F(a, b)=\sum_{x=0}^{M-1} \sum_{y=0}^{N-1} f(x, y) \times e^{-j 2 \pi(u x+v y) / N}$

where the exponential term is the basis function corresponding to each point $F(a, b)$.

To extract defect features, seven textural features are calculated from the weft and warp diagrams of the Fourier spectrum of the fabric images. The vertical direction $f_{y}$ and the horizontal direction $f_{x}$ contain the information about weft and warp yarns, respectively. Seven textural features are calculated as follows:

$P_{1}=|F(0,0)|$

$P_{2}=\left|F\left(f_{x 1}, 0\right)\right|$

$P_{3}=f_{x 1}$

$P_{4}=\sum_{f_{x i}=0}^{f_{x i 1}}\left|F\left(f_{x i}, 0\right)\right|$

$P_{5}=\left|F\left(0, f_{y 1}\right)\right|$

$P_{6}=f_{y 1}$

$P_{7}=\sum_{f_{y i}=0}^{f_{y 1}}\left|F\left(0, f_{y i}\right)\right|$

where $P_{1}$ denotes the image average light intensity. $P_{2}, P_{3}$ and $P_{4}$ represent the changes in the warp or vertical direction. $P_{5}, P_{6}$ and $P_{7}$ represent the changes in the weft or horizontal direction [18]. All features are combined into a single feature vector. This feature vector is used as an input to ANN classifier

\subsection{Wavelet Transform}

The second feature extraction method is the wavelet transform. Wavelet transform is a method of multi-resolution time-frequency analysis [19]. Wavelet coefficients can be modeled by probability density functions (PDF), denoted by $p\left(X ; \theta_{i}\right)$ where $X$ is the wavelet coefficient at the $i$ th subband, $\theta_{i}$ is a set of model parameters. The marginal histogram density of subband coefficients of wavelet transform can be obtained by two parameters of the generalized Gaussian density (GGD) [10]. Generalized Gaussian density function is defined as:

$p(x ; \alpha, \beta)=\frac{\beta}{2 \alpha \Gamma(1 / \beta)} e^{-(|x| / \alpha)^{\beta}}$

where $\Gamma($.$) is the Gamma function., i.e.,$ $\Gamma(h)=\int_{0}^{\infty} e^{-t} t^{h-1} d t, h>0$.

The $\alpha$ parameter of Eq.(10) denotes the standard deviation of PDF peak, while $\beta$ denotes the decreasing rate of the PDF peak. $\alpha$ and $\beta$ parameters are estimated using maximum-likelihood estimator. Thus, an estimator algorithm is required. Do and Vetterli [18] used the maximumlikelihood (ML) estimator. ML estimator can be described as follows:

Let us describe the likelihood function of the given sample distribution $x=\left(x_{1}, x_{2}, \ldots, x_{L}\right)$ having independent component as:

$L(x ; \alpha, \beta)=(x ; \alpha, \beta)=\log \prod_{i=1}^{L} p\left(x_{i} ; \alpha, \beta\right)$

In Eq.(11), $\alpha$ and $\beta$ are parameters of GGD. These parameters can be estimated by the likelihood equation. An efficient estimation of $\alpha$ and $\beta$ using Newton-Raphson iterative procedure presented in [19]. The PDF of each wavelet subband can be calculated via $\alpha$ and $\beta$ parameters. The PDF function consists of dominant textural features. In this paper, we apply wavelet transform-based defect detection method using maximum-likelihood estimator to estimate the $\alpha$ and $\beta$ parameters. After the feature vector is calculated, ANN classifier is trained to classify the fabric defects. The trained neural network is used for real-time defect classification.

\subsection{Shearlet Transform}

The wavelet transform is not a convenient analysis for high dimensional data, and it has less information about the multi-direction analysis of image. Shearlet transform is a multiscale mathematical framework for image analysis [14]. Shearlet transform provides more multiscale and multidirectional geometrical information of multidimensional data. Compared to the traditional wavelet and Fourier transforms, shearlet transform can extract most discriminating features such as orientations in images. The frequency supports of some vertical and horizontal shearlets are showed in Figure 2. Given the shear parameter $s(s \in R)$, the translation parameter $t\left(t \in R^{2}\right)$ and scaling parameter $a(a>0)$, the shearlet function is described as [8]: 
$\psi_{a, s, t}=a^{-3 / 4} \psi\left(D_{a, s}^{-1}(x-t)\right)$

where $D_{a, s}^{-1}=\left[a,-a^{1 / 2} ; 0, a^{1 / 2}\right]$.

The mother shearlet function $\psi$ is defined as $[8,14]$ :

$\psi\left(\xi_{1}, \xi_{2}\right)=\psi_{1}\left(\xi_{1}\right) \psi_{2}\left(\frac{\xi_{2}}{\xi_{1}}\right)$

where $\psi_{1}$ denotes a wavelet and $\psi_{2}$ denotes a bump function. The continuous shearlet transform is defined by [14]:

$$
S H_{\psi} f(a, s, t)=\left\langle f, \psi_{a_{1}, t, t}\right\rangle
$$

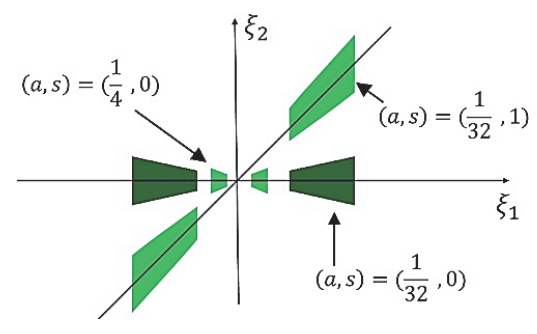

Figure 2. Frequency support of shearlets for different values of $a$ and $s$.

\section{THE PROPOSED DST-PCA APPROACH}

\subsection{Calculation of features}

Multi-scale frequency decomposition of the image produces many different subbands. In order to effectively extract the textural features, this high-dimensional subband space is reduced using different methods:

1) Statistical features: the statistical features can be computed from spatial distribution of gray values. Statistical features such as mean, variance, standard deviation, skewness, moment, are used to characterise the histograms between normal and defective fabric images. They are useful and easy to be calculated. In this paper, means and variances of subbands are used.

2) Geometrical features: the geometric features encode geometric informations such as edge, shape and other landmarks in images. They can extract local geometric information. However, the geometric features are not useful for the fabric images because they need a segmentation step.

3) Textural features: the textural features are useful informations including the spatial arrangement of the image pixels. Fourier transform, wavelet transform and GLCM are conventional approaches of textural feature extraction.

In this paper, statistical modelling of shearlet subbands is used for defect classification. Means and variances are computed from directional subbands obtained from the shearlet transform of the fabric image.

\subsection{DST-PCA}

A high-dimensional feature vector is obtained by combining all the features extracted from shearlet subbands. Especially, the size of the resulting shearlet features increases with increasing decomposition scale. In our work, we applied shearlet transform on each fabric image and obtain the shearlet subband coefficients. Shearlet transform decompose an image into $2 \times 2^{n_{j}}+1 \quad(j$ is the decomposition level and $n_{j}$ shows the direction parameter ndir) directional subbands in the vertical and horizontal cones at any scale. In this paper, 6 decomposition levels were used. For each decomposition level, we used 4, 8, 16 and 32 rotations. Shearlet transformation produced 121 multiple directional subbands. Secondly, the means and variances of all directional subband images were computed. This feature vector has the ability of directional sensitivity at different scales. However, some information with irrelevant and correlation may exist in the shearlet transform-based vector. This situation leads to both computational complexity and wrong defect recognition. Therefore, we need to use a feature vector with less computational cost. Dimension reduction can be used to remove features with irrelevant and correlation.

Principal Component Analysis (PCA) is used as a technique of dimension reduction. PCA can project high-dimensional features into a low-dimensional feature subspace by orthogonal linear transformation. Also, PCA simplifies the description of the feature set. By using top $k$ eigenvectors which have larger eigenvalues for subspace approximation, PCA provides a lower dimension projection to emphasize the underlying fundamental structures of the highdimensional feature space [15]. In order to achieve these goals, PCA calculates new variables called principal components which are obtained as linear combinations of the original variables. The components are calculated from the Singular Value Decomposition (SVD) of the feature data set. The ranking of the principal components in order of their significance is represented by the eigenvalues associated with the vector for each principal component. The significance of principal components is related to the variability in the data they capture. More theoretical analysis about PCA method has been explored in [20].

A feature descriptor called DST-PCA is developed to eliminate the shortcomings of the shearlet transform. Our descriptor improves the performance of traditional shearlet transform. The proposed DST-PCA method includes the following major steps:

Step 1 Fabric images are normalized to have zero mean and unit standard variance. The normalization of fabric images provides invariance to linear changes of illumination.

Step 2 Images are decomposed by shearlet transform into many subbands and then coefficient matrices of subbands are computed in both horizontal and vertical cones at different scales.

Step 3 The means and variances are calculated from all coefficient matrices of subbands computed in Step 2, and a high-dimensional feature vectors are constructed.

Step 4 The low-dimensional feature vector is built by PCA method.

Step 5 An artificial neural network (ANN) classifier is employed to perform classification. 


\section{EXPERIMENTAL RESULTS}

The experiments were implemented as follows: In non-realtime experiments, versatile tests were performed. Firstly, we applied shearlet transform on each fabric image and obtain the shearlet subband coefficients. Shearlet transform decompose an image into $2 \times 2^{n_{j}}+1 \quad(j$ is the decomposition level and $n_{j}$ shows the direction parameter ndir) directional subbands in the horizontal cone and vertical cone at any scale, respectively. In this paper, 6 decomposition levels were used. For each decomposition level, we used 4, 8, 16 and 32 rotations. Secondly, the means and variances of all directional subbands were computed and PCA approach was used to reduce size of the feature vectors. Finally, the feature vectors of fabric database were fed into the back propagation ANN classifier for image classification.

The Fourier transform, wavelet transform modeled by a generalized Gaussian density (Wavelet-GGD) and GLCM methods are used to compare with our proposed approach. We evaluate the performances in terms of classification accuracy and size of feature vector

The general architecture of the ANN consists of input layer, a single hidden layer and the output layer, as shown in Figure 3. ANN is trained using Levenberg-Marquadt (LM) algorithm. A Sigmoidal transfer function is used in the hidden layer. A linear transfer function is used in the output layer. The number of nodes in the input layer depends on the dimensionalities of the input pattern. The number of neurons in hidden layer are determined as 20. The output layer contains one node corresponding to normal or defective fabric.

\subsection{Non-real time experiments}

A comprehensive database (i.e., FDDD) was used in nonreal time experiments. The performance of DST-PCA was compared with results of three feature extraction approaches. For Fourier transform, seven statistical features of the Fourier power spectrum were extracted, including means, standard deviation, variance, coefficient of variation, skewness, kurtosis and moment. These parameters were used to define the histograms of normal and defective fabrics. In the wavelet transform, we used the traditional pyramid wavelet decomposition with three levels using the Daubechies orthogonal filters $\left(D_{4}\right.$ filters). From a single image in the fabric database, two GGD parameters were calculated from each of nine wavelet subbands using the ML estimator defined in the Section 3.2.

Table 1 shows the comparison of the classification rates. It is obvious that the highest classification rate is obtained when using DST-PCA method. This is because the shearlet transform can capture the intrinsic geometrical features of the texture and has a better approximation rate than the wavelet transform. Moreover, it provides a high degree anisotropy and multi-scale directionality. Since Fourier and wavelet transforms have limited multi-directional sensitivity, they fail to effectively extract the multi-directional features.

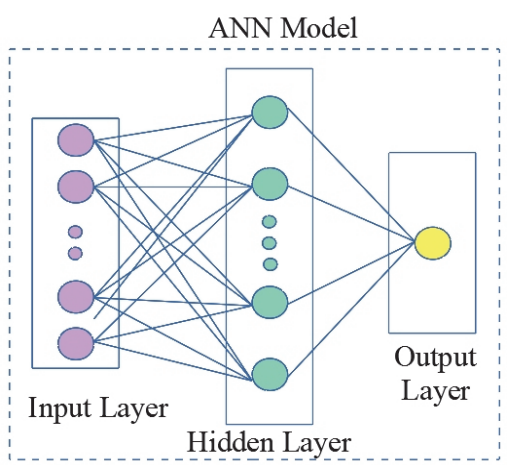

Figure 3. Scheme of the architecture of the used ANN

To evaluate the classification accuracy, the ROC curve is used. A ROC curve is generated from true positive rate versus false positive rate by varying the threshold values [21]. Threshold values are calculated with a decision function. Figure 4 shows the ROC curves for different texture features by ANN classifier. It is obvious that the accuracy of DST-PCA is better than those of other approaches. The ROC curve of the DST-PCA is more convex than others, which means that the area under the ROC curve of the proposed approach is greater than those of other approaches.

Table 1. Classification rates with different texture analysis methods

\begin{tabular}{|l|l|l|l|}
\hline Method & Combined features & $\begin{array}{l}\text { Feature } \\
\text { dimensions } \\
\text { rate (\%) }\end{array}$ & $1 \times 236$ \\
\hline GLCM & Contrast, energy, correlation and homogeneity & 93.00 & $1 \times 7$ \\
\hline Fourier & $\begin{array}{l}\text { Mean, standard deviation, variance, coefficient of variation, skewness, kurtosis and } \\
\text { moment }\end{array}$ & 94.60 & $1 \times 18$ \\
\hline Wavelet-GGD & Standard deviation of PDF peak, decreasing rate of the PDF peak & 90.55 & $1 \times 24$ \\
\hline DST-PCA & Mean and variance & 95.46 & $1 \times$ \\
\hline
\end{tabular}
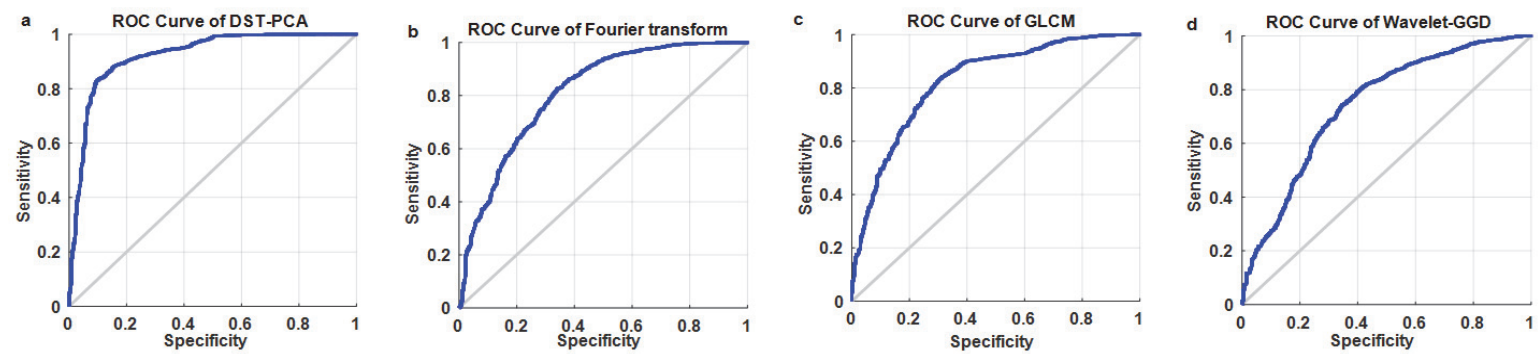

Figure 4. ROC analysis of different feature based detection 
In order to quantitatively compare the performance of defect detection results with different approaches, five different indexes have been used [22]. These indexes are named as sensitivity, specificity, accuracy, negative predictive value (NPV) and positive predictive value (PPV). The definitions of five common indexes are given by:

$$
\begin{aligned}
& \text { Sensitivity }=\frac{T P}{T P+F N} \\
& \text { Specificity }=\begin{array}{c}
T N \\
T N+F P
\end{array} \\
& \text { Accuracy }=\frac{T N+T P}{T N+F N+T P+F P}
\end{aligned}
$$

$$
\text { Positive predictive value }=\frac{T P}{T P+F P}
$$

where $\mathrm{FN}$ is the misclassification rate of defected fabrics; TP denotes the correct classification rate of defected fabrics; FP denotes the misclassification rate of defect-free fabrics and TN is the correct defect-free fabrics.

The training of ANN classifier is a time-consuming work. A training stage is necessary to extract the suitable and reliable training parameters from non-real time experiments to detect the fabric defect in real time defect detection. Thus we obtain a trained ANN model by use of fabric database. This ANN model has been used for the real-time fabric inspection. Therefore, our real-time inspection procedure implements only the feature extraction and ANN testing steps.

\subsection{Real-time experiments}

To detect fabric defect, we developed a user-friendly program interface in Matlab 2014b program. This program also benefits from the Matlab image processing toolbox. Our program automatically detects whether or not the fabric image is defected. The main window interface of the developed fabric defect detection program is shown in Figure 5. The proposed DST-PCA approach has been implemented on a circular knitting machine. Line-scan camera is mounted on the knitting machine over the fabric surface. The developed vision prototype is shown in Figure 6(a). Real-time experiments are executed on Intel Core i7, $3.5 \mathrm{GHz} \mathrm{CPU}$ with $16 \mathrm{~GB}$ RAM. The architecture of the experimental system is given in Figure 6(b).

Our defect detection system contains two algorithmic modules, the feature extraction module and the defect classification module. In the feature extraction module, textural features are extracted by using the DST-PCA and other approaches. For defect classification, we use a threelayer ANN [23]. As mentioned in Section 2, each of feature extraction approaches has been implemented independently on the circular knitting machine. During the production of the fabric on the circular knitting machine, four different fabric defects have been produced by using fabric operator. For real-time defect detection, circular knitting machine has been operated. The vision prototype acquires fabric images continuously using one line-scan. Firstly, the acquired fabric images are normalized. After this process, the DST-PCA procedure is carried out for feature extraction. In similar way, methods similar to DST-PCA have been applied to fabric inspection.

The feature vectors are used as an input for ANN classifier. The weighting coefficients of ANN model have been obtained during the non-real time experiments. Therefore, the ANN training is not performed. It is found that our realtime defect detection prototype is capable of identifying the four fabric defects.

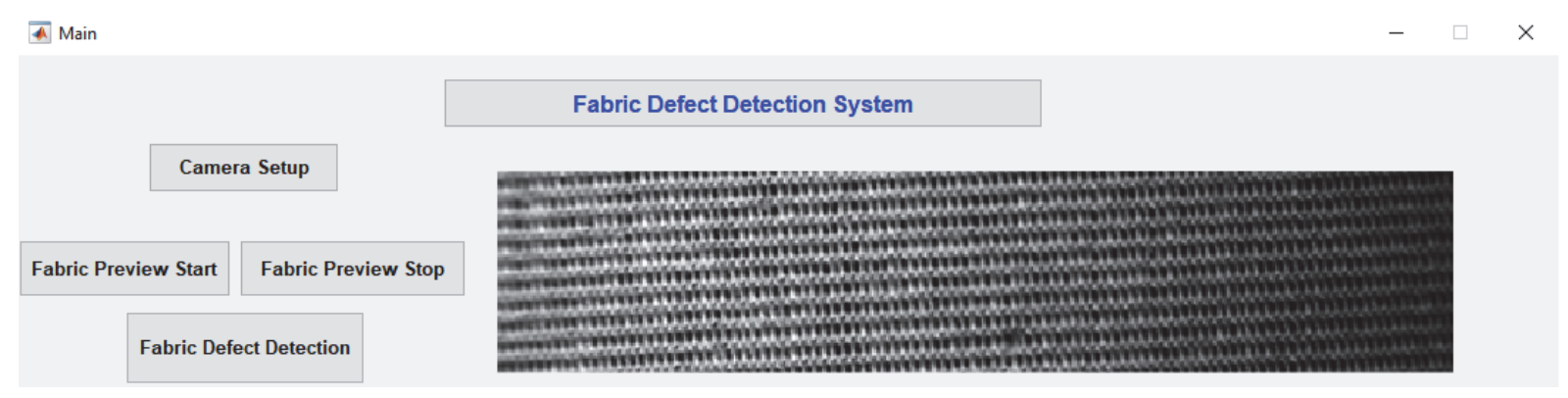

Figure 5. Snapshot of the developed fabric defect detection program window

a

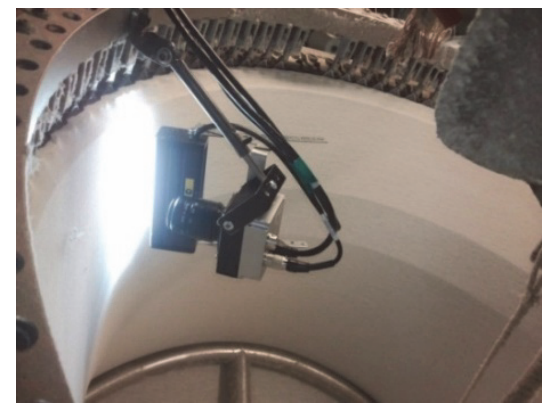

b

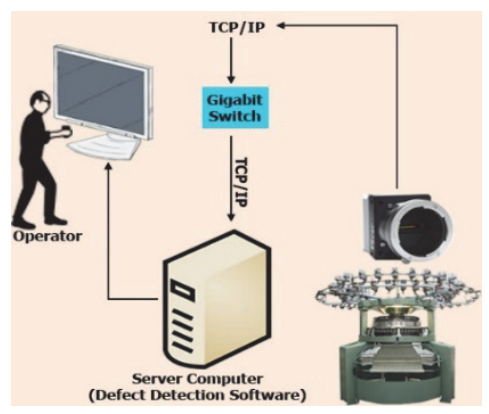

Figure 6. (a) Layout of line-scan camera and line light for fabric defect detection on circular knitting machine, (b) Layout of inspection system of knitting fabric defects 
The concept of implementing shearlet transform looks promising to detect common defects of single jersey fabric. However, some defect types such as rib, lacoste and ottoman cannot be classify by our method. The quantitative results of classification sensitivity, specificity, accuracy, NPV and PPV for different texture analysis approaches are presented in Table 2. All quantitative results confirmed that DST-PCA approach is better than those of other feature extraction methods.

We tested all methods and calculated an average classification rate for four defect types according to real-time implementation. These defects were obtained from the same kind of single jersey knitted fabric in the real-time experiment. Figure 7 denotes that the average classification rate of DST-PCA is greater than for other methods. The detection results obtained during the evaluation period show that a robust real-time defect detection system for circular knitting machine can be created.

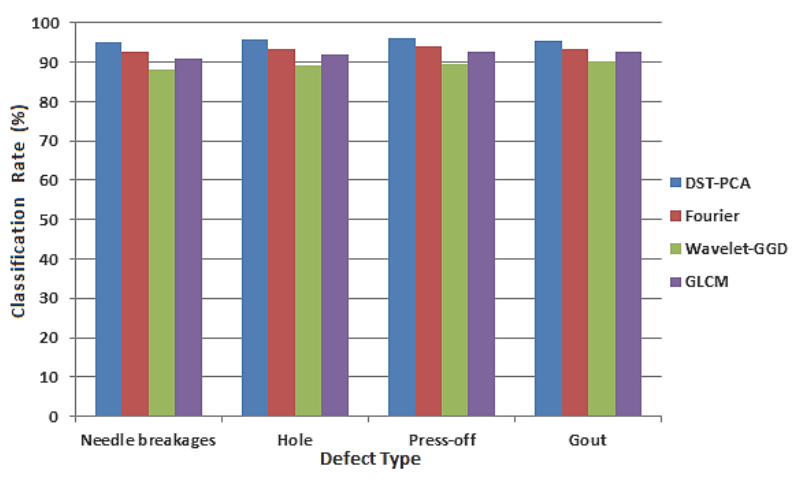

Figure 7. Comparison of detection ratio according to different defect types

\section{CONCLUSIONS}

In this paper, we have presented a computer vision-aided defect detection system to detect circular knitting fabric defects using shearlet transform. The proposed system has been successfully implemented to detect defects on a circular knitting machine. It should be noted that real-time experiments have only been performed on a circular knitting machine. The comparative experiment results indicated that the proposed shearlet-based features could more characterize fabric defects than other three existing methods. It has high defect detection accuracy, sensitivity, specificity, and a low rate of false alarms. On the other hand, there are some weaknesses on the DST-PCA method. There are still some undetected fabric defects such as structural and color defects on single jersey knitted fabric. Therefore, in the future work, it is planned to pay more attention on these defect types. On the other hand, our defect detection system cannot be used simultaneously on several circular knitting machine connected to this system together. Future works will eliminate the shortage of the system in this direction.

\section{ACKNOWLEDGMENT}

This work was supported by the Ministry of Science, Industry and Technology, Turkey (0127.STZ.2013-1).

Table 2. Summary of the quantitative results for different texture features using ANN (unit: \%)

\begin{tabular}{|l|l|l|l|l|}
\hline \multirow{2}{*}{ Index } & \multicolumn{4}{l}{ Feature extraction methods } \\
\cline { 2 - 5 } & DST-PCA & Fourier & Wavelet-GGD & GLCM \\
\hline Accuracy & $\mathbf{9 4} \pm \mathbf{4 . 2}$ & $93 \pm 3.8$ & $88 \pm 6.4$ & $91 \pm 2.3$ \\
\hline Sensitivity & $\mathbf{9 5} \pm \mathbf{4 . 3}$ & $93 \pm 5.1$ & $89 \pm 5.9$ & $92 \pm 3.8$ \\
\hline Specificity & $\mathbf{9 3} \pm \mathbf{5 . 0}$ & $91 \pm 6.1$ & $91 \pm 4.9$ & $90 \pm 3.1$ \\
\hline PPV & $\mathbf{9 2} \pm \mathbf{6 . 2}$ & $91 \pm 6.4$ & $89 \pm 6.3$ & $90 \pm 3.8$ \\
\hline NPV & $\mathbf{9 4} \pm \mathbf{4 . 8}$ & $92 \pm 5.1$ & $87 \pm 7.5$ & $91 \pm 4.2$ \\
\hline
\end{tabular}

\section{REFERENCES}

1. Hanbay K., Talu M. F., Özgüven Ö F., Öztürk D., 2015, Fabric defect detection methods for circular knitting machines, In: 23nd Signal Processing and Communications Applications Conference (SIU), p:735-738

2. Raheja J.L., Ajay B., Chaudhary A., 2013, Real time fabric defect detection system on an embedded DSP platform, Opt. Int. J. Light Electron. Opt., V.124, No.21, p: 5280-5284

3. Hanbay K., Talu M. F., Özgüven Ö F., 2016, Fabric defect detection systems and methods—A systematic literature review, Opt. Int. J. Light Electron. Opt., V.127, No.24, p:11960-11973

4. Salem, Y., Nasri, S., 2010, Automatic recognition of woven fabrics based on texture and using svm, Signal Image Video Process., V.4, No.4, p:429-434

5. Ismail N., Syahrir W.M., Zain J.M., Hai T., 2011, Fabric authenticity method using fast Fourier transformation detection, 2011, International Conference on Electrical, Control and Computer Engineering (INECCE), p:233-237

6. Hanmandlu M., Choudhury D., Dash S., 2015, Detection of defects in fabrics using topothesy fractal dimension features, Signal Image and Video Processing, V.9, No.7, p:1521-1530

7. Li Y., Ai J., Sun C., 2013, Online Fabric Defect Inspection Using Smart Visual Sensors, Sensors, V. 13, No. 4, p:4659-4673

8. Tsai D., Huang T, 2003, Automated surface inspection for statistical textures, Image Vis. Comput., V.21, p:307-323 
9. Hu G.H., Wang Q.H., Zhang G.H., 2015, Unsupervised defect detection in textiles based on Fourier analysis and wavelet shrinkage, Appl. Opt., V.54, p: 2963-2980

10. Karlekar V. V., Biradar M. S., Bhangale K. B., 2015, Fabric defect detection using wavelet filter, International Conference on Computing Communication Control and Automation, p: 712-715

11. Kumar A., 2003, Neural network based detection of local textile defects, Pattern Recognit., V. 36, p: 1645-1659

12. Kang Z., Yuan C., Yang Q., 2013, The fabric defect detection technology based on wavelet transform and neural network convergence, IEEE International Conference on Information and Automation (ICIA), p: 597-601.

13. Zhang Y., Lu Z., Li J., 2010, Fabric defect classification using radial basis function network, Pattern Recognition Letters, V. 31, No. 13, p: 2033-2042,

14. Liu X., Mei W., Du H., Bei J., 2016, A novel image fusion algorithm based on nonsubsampled shearlet transform and morphological component analysis, Signal Image Video Processing, V.10, No.5, p:959-966

15. Sengur A., 2008, An expert system based on principal component analysis, artificial immune system and fuzzy kk-NN for diagnosis of valvular heart diseases, Computers in Biology and Medicine, V.38, No.3, p:329-338

16. Hanbay K., Talu M. F., Özgüven Ö F., Öztürk D., 2017, Real time fabric defect detection by using fourier transform, Journal of the Faculty of Engineering and Architecture of Gazi University, V.32 No.1,p:151-158

17. Cooley J.W., Tukey J.W., 1965, An algorithm for the machine calculation of complex fourier series, Mathematics of Computation, V.19, No.90, p:297-301

18. Malek A. S., Drean J. Y., Bigue L., Osselin J. F., 2013, Optimization of automated online fabric inspection by fast Fourier transform (FFT) and crosscorrelation, Textile Research Journal, V.83, No.3, p:256-268

19. Do M.D., Vetterli M., 2002, Wavelet-based texture retrieval using generalized gaussian density and kullback-leibler distance, IEEE Transactions on Image Processing, V.11, No.2, p:146-158

20. Abdi H., Williams L. J., 2010, Principal component analysis, Wiley Interdiscip. Rev. Comput. Stat., Vol.2 No.4, p: 433-459

21. Marrocco C., Duin R.P.W., Tortorella F., 2008, Maximizing the area under the ROC curve by pairwise feature combination, Pattern Recognition, V.41, No.6, p:1961-1974

22. Zhou S., Shi J., Zhu J., Cai Y., Wang R., 2013, Shearlet-based texture feature extraction for classification of breast tumor in ultrasound image, Biomedical Signal Processing and Control, V.8, No.6, p:688-696

23. Kargı V. S. A., 2014, A comparison of artificial neural networks and multiple linear regression models as in predictors of fabric weft defects, Tekstil ve Konfeksiyon, V.24, No.3, p:309-316 